\title{
A MULTIPLICAÇÃO E O ENSINO: UM ESTUDO A PARTIR DAS PROPOSIÇÕES DE DAVYDOV
}

\author{
Maria Luiza Evangelista Gil ${ }^{1}$, Luciana Figueiredo Lacanallo Arrais $^{2}$ \\ ${ }^{1}$ Graduanda do curso de Pedagogia da Universidade Estadual de Maringá - UEM, PR. ORCID iD: \\ https://orcid.org/0000-0002-9477-0326. E-mail: maria.evangelistagil@gmail.com \\ ${ }^{2}$ Doutora em Educação pela Universidade Estadual de Maringá -UEM. Professora adjunta do Departamento de Teoria \\ e Prática da Educação da Universidade Estadual de Maringá - DTP/UEM e do Programa de Pós-Graduação em \\ Educação - PPE/UEM. ORCID iD: https://orcid.org/0000-0001-5297-7823. E-mail: Iflacanallo@uem.br
}

\section{RESUMO}

O objetivo deste artigo é discutir o processo de ensino da multiplicação, tendo como princípios os pressupostos da Teoria Histórico-Cultural - THC. A matemática está presente no nosso dia a dia e permite a comunicação, a resolução de problemas e a promoção de relações sociais, o que evidencia a importância e a necessidade da apropriação dos seus conceitos. Portanto, por meio de uma pesquisa bibliográfica, foram feitos estudos sobre Vygotsky, Davydov, Moura, Rosa, Galdino e outros autores contemporâneos ligados a essa mesma perspectiva, na busca da compreensão de princípios que nos auxiliem a ensinar a multiplicação nos anos iniciais de escolarização. Constatamos que o ensino precisa ir além dos aspectos empíricos em direção ao desenvolvimento do pensamento teórico, proporcionando a execução de tarefas e ações que façam com que o aluno entre em atividade de estudo e compreenda a essência que caracteriza o conceito. A pesquisa indica que o tema precisa ser mais investigado no intuito de que se tenham mais proposições e encaminhamentos para organizar a aprendizagem da multiplicação nos anos iniciais de escolarização.

Palavras-chave: Ensino da multiplicação. Davydov. Organização do ensino. Teoria Histórico-Cultural.

\section{CONCEPT OF MULTIPLICATION IN DAVYDOV'S PROPOSITIONS: REVIEWING THE EDUCATIONAL ORGANIZATION}

\begin{abstract}
The objective of this article to discuss the process of teaching multiplication, having as principles the assumptions of the Historical-Cultural Theory - THC. Mathematics is present in our daily life and allows us to communicate, solve problems and promote social relations. This proves the importance and need for the appropriation of the concepts involved in the aritmetic operations is essential, especially multiplication. Therefore, through a bibliographical research, studies were made on Vygostki, Davydov, Moura Rosa, Galdino and other contemporary authors linked to this same perspective, the search for understanding principles that help us teach multiplication in the early years of schooling. We found that teaching needs to go beyond empirical aspects towards the development of theoretical thinking, being indispensable the execution of tasks and actions that make the student enter into study activity and understand the concept. The research shows that the theme needs more investigated in order to have more propositions and referrals to ensure the learning of the multiplicative concept.

Keywords: Teaching multiplication. Davydov. Teaching organisation. Historical-Cultural Theory.

\section{CONCEPTO DE MULTIPLICACIÓN EN LAS PROPUESTAS DE DAVYDOV: REVISIÓN DE LA ORGANIZACIÓN EDUCATIVA}

\section{RESUMEN}

El objetivo de este artículo es discutir el proceso de enseñanza de la multiplicación, teniendo como principios los presupuestos de la Teoría Histórico-Cultural - THC. Las matemáticas están presentes en nuestra vida cotidiana y permiten la comunicación, la resolución de problemas y la promoción de las 
relaciones sociales, lo que pone de relieve la importancia y necesidad de la apropiación de sus conceptos. Por lo tanto, a través de una investigación bibliográfica, se realizaron estudios sobre Vygotsky, Davydov, Moura, Rosa, Galdino y otros autores contemporâneos enlazados a esta misma perspectiva, en la búsqueda de la comprensión de principios que nos ayuden a enseñar la multiplicación en los primeros años de escolaridad. Encontramos que la enseñanza necesita ir más allá de los aspectos empíricos hacia el desarrolo del pensamiento teórico, proporcionando la ejecución de tareas que llevaron al estudiante a entrar en la actividad de estudio y compreender la esencia que caracteriza el concepto. La investigación indica que el tema necesita ser investigado más a fondo con el fin de tener más proposiciones y referencias para organizar el aprendizaje de la multiplicación en los primeiros años de escolaridad.

Palabras clave: Enseñanza de la multiplicación. Davydov. Organizacion de la enseñanza. Teoria HistóricoCultural.

\section{INTRODUÇÃO}

A matemática vem como resposta à necessidade do homem de controlar quantidades para sobreviver e, com ela, foi e é possível desenvolver funções mentais superiores essenciais à vida em sociedade a partir de um ensino organizado e sistematizado. Mesmo antes de a criança ingressar na escola, a matemática está presente em sua vida, todavia esses conhecimentos não são suficientes para que desenvolva seu máximo potencial humano.

Davydov (1982) destaca que o papel da escola é promover a formação omnilateral do homem e esta só pode ser promovida por meio dos conhecimentos científicos. Oliveira et al. (2017) complementa que a escola precisa ser capaz de fornecer aos alunos a aquisição sistemática dos conhecimentos acumulados na história humana, dentre eles, os matemáticos. Ao constatar que o ensino dessa ciência voltava sua ênfase apenas para elementos sensoriais e reduzia os conceitos ao aspecto empírico, o autor afirmou que um novo sistema de ensino precisaria ser proposto (DAVYDOV, 1987).

Objetivando organizar um ensino direcionado ao desenvolvimento psíquico do sujeito, Davydov (1982) propõe a inter-relação entre os campos aritmético, geométrico e algébrico para ensinar a matemática. Reconhecer as relações entre esses campos e as grandezas seria um princípio em direção à formação do pensamento teórico na escola.

De acordo com Moura et al. (2012), a matemática é um conhecimento organizado que faz parte do contexto histórico vivenciado pela humanidade e que se mantém ao longo dos tempos em contínuo processo de transformação. Ao considerar essa dimensão, tanto os homens primitivos como os contemporâneos têm a necessidade da utilização da linguagem matemática e da apropriação desses conceitos para a vida em sociedade.

Dentre os conceitos matemáticos, destaca-se a multiplicação, isso porque, segundo Rosa (2012), é uma das operações básicas da aritmética que implica em multiplicar sucessivas vezes um mesmo número, a fim de se chegar a um resultado ou produto quando se deseja calcular grandes quantidades. Desse modo, a multiplicação torna possível dobrar, triplicar, quadruplicar e assim sucessivamente grandes quantidades. Dominar essa operação faz parte do conjunto de conhecimentos considerados mínimos até o 5o ano do Ensino Fundamental. No entanto, os alunos não vêm demonstrando aprendizagem das operações de multiplicação e divisão até a conclusão desse período escolar e, de acordo com Ortigão e Junior (2016), isso pode ser verificado tanto pela média de reprovação dos alunos na disciplina de matemática quanto pelas avaliações externas de desempenho escolar.

$\begin{array}{ccc}0 & \text { desempenho dos alunos na } \\ \text { matemática } & \text { especificamente não tem }\end{array}$
satisfatório, pois "a média em Matemática retrai cerca de 26 pontos em uma reprovação e aproximadamente 86 pontos se a reprovação ocorreu por duas vezes ou mais ao longo de sua vida escolar" (ORTIGÃO; JUNIOR, 2016, p. 6). A multiplicação é trabalhada, ainda hoje, nas escolas, de forma empírica e mecânica, o que torna o conteúdo vazio de sentido (ROSA, 2012).

A Teoria Histórico-Cultural (THC) nos mostra que é necessário compreender a essência dos conceitos e superar a dimensão empírica que os caracteriza, pois

o pensamento teórico é uma forma de 
conhecimento convertido em instrumento psicológico (ação mental) que possibilita, por sua vez, lidar com outros conhecimentos. Ou seja, os conhecimentos teóricos representam as máximas potencialidades construídas

historicamente; apropriarse deles e, igualmente, apropriar-se dessa riqueza humana, direito de todos [...] (CATANANTE; ARAUJO, 2014, p. 51).

Assim, é preciso identificar os princípios envolvidos no conceito de multiplicação buscando compreendê-los além do empírico, para proporcionar a formação do pensamento teórico. Para isso, o professor propõe tarefas e ações que façam com que o aluno entre em atividade de estudo, possibilitando 0 desenvolvimento de funções indispensáveis ao homem, como a abstração e a generalização.

Diante disso, o objetivo desta pesquisa é discutir o processo de ensino da multiplicação, tendo como princípios os pressupostos da Teoria Histórico-Cultural. Por meio de uma pesquisa bibliográfica, , apresentamos o conceito de multiplicação nas obras de Davydov e seus colaboradores. $\mathrm{Na}$ sequência, discutimos as implicações desses princípios para o ensino nos anos iniciais do Ensino Fundamental, buscando organizar a aprendizagem da multiplicação nessa etapa da escolarização.

\section{A MATEMÁTICA COMO LINGUAGEM}

A educação escolar é essencial à formação do homem, pois possibilita que ele aprenda e se desenvolva. De acordo com Davydov (1982), para possibilitar o aprendizado e promover o desenvolvimento das funções psíquicas superiores próprias do ser humano a escola é indispensável. Segundo Oliveira et al. (2017) a educação se relaciona ao trabalho não material, pois seu objetivo é produzir conhecimento e cultura, transmitindo para as gerações mais novas.

Porém, para isso a escola precisa organizar o ensino, a fim de assegurar que todos se apropriem do conhecimento científico essencial à vida em sociedade, pois "o conhecimento mais elaborado, 0 saber sistematizado e científico desenvolve as funções psíquicas em suas máximas possibilidades e engendra o pensamento crítico e conceitual" (OLIVEIRA et al., 2017, p. 62). Um desses conhecimentos é a matemática, mas, que para ensiná-lo o professor precisa ter claro o significa e sua função no ensino para que possamos definir princípios e estratégias para conduzi-lo.

$\mathrm{Na}$ sociedade, é pela linguagem que os homens estabelecem relações entre si e entre os objetos, de modo que a interação social seja possível. Sabemos que existem diversas formas de linguagem que permitem o desenvolvimento, a interação e a comunicação em sociedade: a fala, a escrita, a literatura, a música, os números, dentre tantas outras.

A partir disso, Vygotsky (2001) afirma que a linguagem é um sistema constituído de signos importantes para os humanos e permite mudanças em seus processos psíquicos, possibilitando que o homem trabalhe com objetos, realize análises, abstrações, generalizações e a comunicação em si. A linguagem garante a transmissão, a preservação e a assimilação de experiências e conhecimentos acumulados ao longo do tempo, portanto "a linguagem é antes de tudo, um meio de comunicação social, de enunciação e compreensão" (VYGOTSKY, 2001, p. 11).

Dentre as muitas linguagens, a matemática é para a THC uma delas. Enquanto linguagem, a matemática auxilia as pessoas a solucionarem problemas, promoverem relações, comunicarem-se entre si e controlarem quantidades.

A matemática é um destes instrumentos que capacitam o homem para satisfazer a necessidade de relacionar-se para resolver problemas, em que os conhecimentos produzidos a partir dos problemas colocados pela relação estabelecida entre os homens e com a natureza foram-se especificando em determinados tipos de linguagem que se classificaram como sendo matemática (MOURA, 2007, p. 48).

Assim sendo, a matemática é um 
instrumento simbólico que permite ao homem satisfazer suas necessidades integrativas, motivadas pelo entorno, promovendo $\mathrm{o}$ desenvolvimento de capacidades para medir, contar, explicar, entre outros. Portanto, segundo Moura (2007, p. 50), "o desenvolvimento do conhecimento matemático, nesse processo, é parte da satisfação da necessidade de comunicação entre os sujeitos para a realização de ações colaborativas." De acordo com o autor, ela possibilita enfrentar diversos problemas na sociedade, a satisfação coletiva e individual das necessidades humanas.

Historicamente, essa linguagem foi criada para satisfazer às necessidades do homem, sendo a matemática uma produção que "[...] estuda o mundo material de um ponto de vista singular, seu objetivo imediato são as formas espaciais e as relações quantitativas do mundo" (DAVYDOV, 1982, p. 106, tradução nossa ${ }^{1}$ ). Para Rosa (2012), há pelo menos cinco mil anos, a matemática vem ao encontro da necessidade que temos de determinar relações, promover comunicação e solucionar dilemas.

Conforme pesquisa feita por Fiorentini (1995), no Brasil, a partir da década de 1920, diversas vertentes buscaram compreender a matemática, com base em diferentes entendimentos sobre essa ciência: a formalista clássica, a empírico-ativista, a formalista moderna, a tecnicista, a construtivista e a sócioetnocultural. A vertente formalista clássica baseava-se nas ideias e formas da matemática clássica, no modelo euclidiano e na concepção platônica de matemática, concebendo-a como algo que não foi inventado pelo homem, mas sim preexistia em um mundo ideal. $O$ ensino nessa tendência, de acordo com o autor, era centrado no professor, pois é quem transmitia e expunha o conteúdo, e o aluno era visto de forma passiva, que tinha o objetivo de memorizar e reproduzir o que lhe era transmitido.

A segunda vertente, a empírico-ativista, concebia o aluno como centro no processo de aprendizagem, portanto ele era um ser ativo e o ensino baseava-se em atividades com um material didático rico, com um ambiente que estimulasse e possibilitasse jogos e experimentos de acordo com Fiorentini (1995). A finalidade desses recursos era estimular a criatividade e potencializar os interesses individuais e da

\footnotetext{
${ }^{1}$ Todas as traduções feitas no decorrer do artigo são de responsabilidade das autoras.
}

sociedade. Os materiais foram criados por Maria Montessori, com o objetivo de que os alunos possam manipular os objetos por meio da habilidade sensorial, sendo que a aprendizagem é realizada pelos sentidos e pela experimentação, bem como por meio da repetição dos movimentos realizados com os materiais, por isso esses objetos são exemplos dessa vertente.

A terceira tendência denominada de formalista moderna defendia, de acordo com Fiorentini (1995), um ensino autoritário, centrado no professor, sendo o aluno visto de forma passiva, pois a ênfase centrava-se na dimensão formativa. Já a vertente tecnicista, a quarta tendência, buscava um enfoque em técnicas especiais para o ensino e administração escolar, tendo como prioridade o desenvolvimento de habilidades técnicas, baseando-se no funcionalismo, portanto a escola teria um papel importante na estabilidade e na manutenção do sistema.

A quinta tendência é a construtivista que vê a matemática como uma concepção humana formada por estruturas e relações entre grandezas e formas, por meio da interação do homem com o meio. Defende uma prática pedagógica que construa a estrutura do pensamento matemático por meio de materiais concretos. A sexta vertente é a sócioetnocultural que compreende as diferenças culturais e sociais na sociedade e sua interferência na educação, concebendo a matemática como algo produzido histórico e culturalmente. A finalidade, segundo Fiorentini (1995), é a busca da desmistificação e compreensão da realidade, por isso para esse ensino é preciso buscar uma relação com o cotidiano e a cultura do aluno.

Damazio e Rosa $(2013$, p. 39) ressaltam que esta última tendência também nominada como histórico-cultural compreende que a educação matemática tem o objetivo de direcionar os estudos no processo de concepção desse conhecimento, vendo-a como decorrente das práticas sociais de tal época, como atividades políticas, econômicas e sociais. Essa vertente tem sua base no materialismo histórico-dialético, portanto "[...] busca explicar a vida social pelas mudanças qualitativas das formas especificamente humanas". Para os autores, essa tendência analisa a gênese das mediações, observando as mudanças nas práticas sociais e a relação que o homem estabelece com a natureza, o que é necessário para que aconteça o desenvolvimento histórico e das funções 
psicológicas superiores humanas.

De acordo com essa compreensão, a matemática é um conhecimento histórico em produção, por meio das relações sociais, portanto o aluno aprende ao conferir sentido às ideias matemáticas, sendo a sala de aula um lugar para a interação e a produção de sentidos e significados. Por meio dos conhecimentos matemáticos, o homem pode realizar o controle de quantidades, grandezas e medidas

Temos Vygotski (2001), Davydov (1982, 1988), Rosa (2012) e Moura (2007) como alguns dos principais autores que representam essa tendência. A partir desses estudos, a matemática "é obra de várias culturas e de milhares de homens que, movidos pelas necessidades concretas, constituíram coletivamente a Matemática que conhecemos hoje" (FIORENTINI, 1995, p. 31), portanto trata-se de um conhecimento histórico e coletivo.

Compreender a vida por meio das mudanças realizadas pelo homem, ao recorrer à gênese das mediações e ao cotidiano, é um princípio defendido pela THC conforme Damazio e Rosa (2013) apontam. Portanto, os estudos baseados nessa teoria compreendem a matemática, segundo Rosa (2012, p.21), como "um conhecimento produzido historicamente pelo homem com o objetivo de conhecer, interpretar e transformar a realidade". Dessa forma, ela pode ser organizada de três formas:

- Aritmética: estuda os números e operações numéricas (adição, subtração, multiplicação, divisão...);

- Algébrica: relaciona-se à manipulação formal de operações matemáticas, de equações, relação entre grandeza e unidade;

- Geométrica: trabalha com as formas, tamanho, posição relativa de figuras e propriedade do espaço (comprimento, área...).

Nessa divisão dos conceitos por eixos, vemos que a multiplicação faz parte da aritmética e das sete operações básicas, ou seja, a adição, a subtração, a divisão, a radiciação, a logaritmação e a potenciação, as quais "constituem o conjunto de leis operatórias do cálculo que, juntamente com as propriedades estruturais, são mantidas em todos os campos numéricos" (ROSA, 2012, p. 28).

A contagem, o controle de quantidades e as operações tornaram-se uma necessidade quando o homem estabeleceu relações em que esses conhecimentos eram indispensáveis para garantir sua sobrevivência. Neste trabalho, enfocaremos a multiplicação, por ser uma das quatro operações básicas da aritmética. Para Rosa e Hobold (2017, p. 452) a essência da multiplicação está

na relação r de
multiplicidade entre
grandezas (por meio de
unidades básicas e
intermediárias), cujo
resultado conforma uma
sequência, na qual o
produto posterior é uma
unidade de medida
intermediárias maior que
o anterior. Esta essência é
expressa no modelo
universal a $x \mathrm{n}=\mathrm{a} \times$ ( $\mathrm{n}$-1)
$+\mathrm{a}=\mathrm{c}$.

A necessidade de multiplicar é algo histórico, porque, já "[...] os primitivos utilizavam os dedos ou os riscos para representá-los durante o cálculo [...] (GALDINO, 2016, p. 83), porém nas relações estabelecidas nem sempre as quantidades eram pequenas e isso dificultava e inviabilizava a utilização dos dedos e riscos para resolver contas com números maiores. Desse modo, o homem sentia a necessidade de desenvolver procedimentos para realizar contas e controlar grandes quantidades, pois, segundo o autor, a humanidade percebeu que a contagem um a um tinha suas limitações e imprecisões, e contar por meio de agrupamentos maiores foi a melhor solução.

$\mathrm{Na}$ escola, os alunos utilizam os dedos ou riscos para realizar o controle de muitas quantidades, tal como os primitivos faziam, mas isso torna o processo de resolução mais demorado e com possibilidades de imprecisão. Essas formas de controle caracterizam o conhecimento empírico e não permitem a formação do pensamento teórico. No entanto, percebemos que a multiplicação é utilizada em diversas situações do dia a dia e poderia ser mais explorado nas escolas, com problematizações mais próximas a real necessidade de multiplica, por exemplo, se 10 países disputarem uma competição esportiva com 22 atletas em cada equipe, a contagem um a um levaria tempo e não seria tão eficaz.

Por meio da compreensão da estrutura multiplicativa, o cálculo será desenvolvido e se possibilitará "a superação da necessidade permanente de utilização dos riscos, dedos [...]" (GALDINO, 2016, p. 83), ou seja, ao superar por 
incorporação o conhecimento empírico a formação do pensamento teórico estará sendo possibilitada.

É preciso, de acordo com Davydov (1982), que a escola assegure a apropriação dos conceitos científicos para que os alunos desenvolvam o pensamento teórico e suas funções psicológicas superiores. Esse desenvolvimento poderá ser viabilizado por meio de um ensino intencional e organizado, diferenciando-se daquele apreendido fora da escola, o qual não exige sistematização e organização. A seguir, apresentamos as especificidades que envolvem a multiplicação e as possibilidades para que se possa pensar a organização do seu ensino com base em Davydov (1982) e em ROSA; HOBOLD (2017).

\section{A MULTIPLICAÇÃO E O ENSINO}

A escola tem o objetivo de ensinar a matemática, de maneira que assegure o caráter científico do conteúdo, sendo que, para Davydov (1982), é a escola que deve propiciar essa aprendizagem e possibilitar o desenvolvimento do pensamento teórico. Em seu livro "Tipos de generalización en la enseñanza", o autor critica o ensino tradicional que apresenta os conceitos como um produto pronto e acabado, ao reduzílos a dados sensoriais e a traços comuns identificados apenas pela percepção. Para Davydov (1982, p. 157), "[...] é necessário mostrar francamente às crianças a essência abstrata das matemáticas, inculcar-lhes a faculdade de fazer abstrações e de aproveitar sua força teórica", para que a escola forme o pensamento teórico por meio das tarefas proporcionadas.

Em direção à formação desse pensamento, Rosa (2012) afirma que a multiplicação faz parte das operações essenciais: adição, subtração, multiplicação, divisão, radiciação, logaritmação e potenciação. Multiplicar implica em adicionar um mesmo número sucessivas vezes, para se chegar a um produto ou resultado: "[...] $\mathrm{C}=\mathrm{Y} \times \mathrm{A}$. O número de vezes $(Y)$ que a unidade de medida básica $A$ se repete, consiste no total de rosas (C)" (GALDINO, 2016 , p. 71). No esquema apresentado pelo autor, denomina-se de $\mathrm{Y}$ o multiplicador, de $\mathrm{A}$ o multiplicando e de $\mathrm{C}$ o produto, sendo esses os termos dessa operação aritmética.

Nesse sentido, essa operação possui uma relação universal em que $U . X=Y$, sendo que $U$ equivale à unidade intermediária, $X$ corresponde à variável e $\mathrm{Y}$ ao produto, isso porque, mesmo que se mudem os valores, o processo sempre será igual. Rosa e Hobold (2017, p. 451) salientam que "[...] os fatores que a compõem ocupam uma posição definida e conformam uma sequência numérica, na qual cada termo posterior é a soma do anterior, mais uma unidade de medida intermediária, denominada de razão".

Mas, essa compreensão do conceito de multiplicação não é proporcionada pelo ensino tradicional. Davydov (1982), propõe a superação dessa forma de compreensão sugerindo que não se utilize o esquema de percepção representação - conceito para ensinar. De acordo com o autor, esse esquema, primeiro refere-se à compreensão que se tem dos objetos organizados em uma situação singular, como quantidade de flores em vasos, lápis e outros objetos, a representação relaciona-se com a interpretação do objeto que se tem por meio da observação direta dele. Desse modo, a utilização dos dedos ou dos riscos equipara-se à utilização das flores, lápis ou qualquer outro objeto e, assim, forma-se o conceito focalizado na aprendizagem pela reprodução. Torna-se mecânico, uma forma de generalizar e abstrair o conceito que enfatiza apenas o conhecimento empírico.

A Figura 1 reproduz o esquema tradicional de ensino da multiplicação, ou seja, percepção - representação - conceito. Percepção é a compreensão do objeto em si, como vasos, lápis; representação é a utilização de riscos ou dedos para representar o objeto; conceito (formação do conceito).

Figura 1. Representação feita por um aluno de 3으 ano

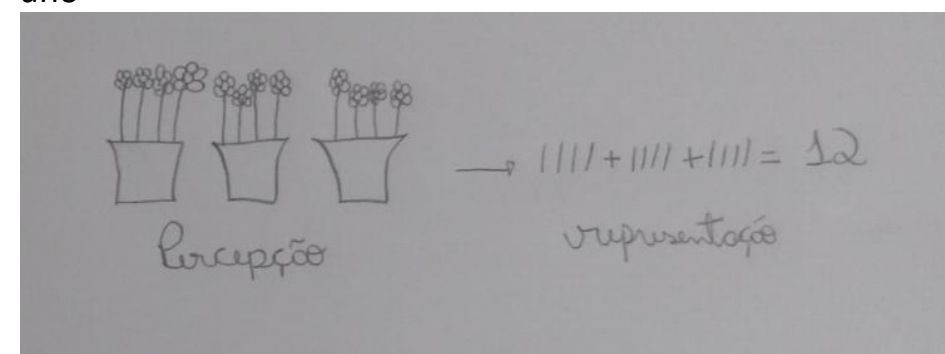

Fonte: das autoras.

Podemos ver essa proposta de resolução em diversos livros didáticos utilizados nas escolas, sendo que essas atividades têm o mesmo sentido, isto é, utilizar a contagem um a um, como proposto pelo esquema do ensino tradicional. Portanto, para Davydov (1982, p. 
155), “[...] crianças em idade escolar especialmente escolas primárias - eles resolvem com sucesso completo basicamente apenas problemas mais do tipo que eles conhecem e cuja identificação anterior é a premissa fundamental para reproduzir o método de resolução concreto que eles assimilaram anteriormente".

O esquema percepção - representação conceito utilizado no ensino tradicional limita o desenvolvimento do aluno e faz com que a aprendizagem se baseie única e exclusivamente na repetição e na memorização, logo o aluno não desenvolve o pensamento teórico, finalidade da escola. Diferente da escola tradicional que promove o desenvolvimento do pensamento empírico, Davydov (1982) propõe uma educação para todos os conceitos com base na abstração generalização - conceito. Isso consiste, primeiramente, em analisar o processo de desenvolvimento daquilo que se estuda, sendo necessário se apropriar do concreto, para que assim seja possível chegar ao entendimento desse por meio da generalização. Esse movimento possibilitará que o aluno se aproprie dos conceitos científicos e desenvolva o pensamento teórico. Abstrações e generalizações sustentam a relação interna entre os objetos e levam à compreensão do conceito.

Distintivamente do conhecimento empírico, o ensino proposto por Davydov (1982) e seus colaboradores para a multiplicação estabelece relações entre os conceitos e propõem que as tarefas que orientam essa aprendizagem do aluno sejam voltadas ao seu desenvolvimento psíquico. De acordo com Davydov (1988), essas tarefas impulsionam o pensamento do aluno e fazem com que a criança busque compreender o conceito, com possibilidades a formação do pensamento teórico. Seguindo esse pressuposto, Rosa et al. $(2016$, p.7) afirma que:

[...] o movimento de apropriação conceitual segue do geral para 0 particular e singular, conduzido pela relação universal. Nele as significações aritméticas, algébricas e geométricas são inter-relacionadas nos procedimentos de redução do concreto ao abstrato (modelação objetal, gráfica e literal), e de ascensão do abstrato ao concreto

(diferentes

aplicações)

Desse modo, o ensino da multiplicação, com base nas pesquisas de Rosa e Hobold (2017), é composto por diversas tarefas de estudo ${ }^{2}$, que partem do concreto que seria a relação direta com o objeto, para depois chegar ao abstrato. Nesse movimento, uma das possibilidades de recursos didáticos a serem adotados, é a reta numérica, que permite $o$ entendimento dos nexos internos da multiplicação. Pela reta, o aluno pode visualizar a unidade intermediária, a variável, o produto e pode realizar a representação gráfica, pois após compreender essa relação universal da multiplicação, trabalhase a memorização, chegando-se ao concreto novamente. Portanto, para se apropriar do conceito de multiplicação, é necessário que o aluno esteja em atividade de estudo ${ }^{3}$, a qual, segundo Davydov (1988, p. 181), possibilita que eles "[...] assimilem o conhecimento no processo de solução autônoma das tarefas, o que lhes permite expor as condições de origem desse conhecimento."

Estar em atividade de estudo, implica no aluno assumir uma ação investigativa com o conceito. Portanto, o professor deve proporcionar tarefas que possibilitem ao aluno abstrair e compreender o conceito de multiplicação. $\mathrm{Na}$ sequência, abordaremos algumas propostas para o ensino da multiplicação que podem proporcionam a formação do pensamento teórico.

\section{Multiplicação e possibilidades de ensino e aprendizagem}

Moura (2001) propõe que uma das formas para organizar o ensino da matemática pode ser a Atividade Orientadora de Ensino (AOE), "[...] aquela que se estrutura de modo a permitir que os sujeitos interajam, mediados por um conteúdo negociando significados, com o objetivo de solucionar coletivamente uma situação-problema [...]" (MOURA, 2001, p. 155). Enquanto uma base teórico-metodológica, a $\mathrm{AOE}$ possibilita que a criança entre em processo de investigação diante de uma situação problema, estimulando-a na elaboração de soluções

\footnotetext{
2 É a unidade do objetivo da ação e das condições para alcançar (DAVYDOV, 1988, p. 178).

${ }^{3}$ Está ligada à elevação considerável do nível ideológico teórico do processo didático educacional na escola primária e contribui para o desenvolvimento do pensamento criativo autônomo dos alunos.
} 
direcionada a compreensão do conceito.

Para materializar a $\mathrm{AOE}$, podemos utilizar jogos, histórias virtuais e situações emergentes do cotidiano, pois proporcionam que o aluno entre em investigação e, dessa forma, além de procurar a resposta para o problema, busca-se também a elaboração de perguntas e argumentos. Portanto, essa proposta pode proporcionar o desenvolvimento do pensamento teórico por meio da compreensão do conceito e não só sua reprodução mecânica.

Nessa direção, algumas ações de estudo precisam ser definidas e Davydov (1988) propõe seis delas, com a finalidade de possibilitar "[...] sua assimilação supõe, por sua vez, a formação nos jovens escolares de abstrações e generalizações que formam a base do pensamento produtivo, o que, em nossa opinião, favorece o desenvolvimento do pensamento teórico em crianças" (DAVYDOV, 1988, p. 189). O autor destaca como ações,

$$
\begin{aligned}
& \text { - transformação de dados } \\
& \text { de tarefas para expor a } \\
& \text { relação universal do } \\
& \text { objeto estudado; } \\
& \text { - modelagem da relação } \\
& \text { diferenciada objetiva, } \\
& \text { graficamente ou por meio } \\
& \text { de letras; } \\
& \text { - transformação do } \\
& \text { modelo de } \\
& \text { relacionamento para } \\
& \text { estudar suas propriedades } \\
& \text { em "forma pura"; } \\
& \text { - construção do sistema de } \\
& \text { tarefas particulares a } \\
& \text { resolver por um } \\
& \text { procedimento geral; } \\
& \text { - controle sobre o } \\
& \text { cumprimento das ações } \\
& \text { anteriores; } \\
& \text { - avaliação da assimilação } \\
& \text { do procedimento geral } \\
& \text { como resultado da solução } \\
& \text { da tarefa de estudo } \\
& \text { (DAVYDoV, 1988, p. 181). }
\end{aligned}
$$

Portanto, essas ações são capazes de auxiliar na formação do pensamento teórico criando condições ao aluno para entre em atividade de estudo e compreenda o conceito. $\mathrm{Na}$ utilização dessas seis ações, o professor coloca um objetivo didático para o aluno (um problema) para que identifique a relação geral do objeto de estudo. Em seguida, com a modelação das relações que foram identificadas de forma concreta, gráfica e literal, o aluno poderá compreender a relação universal que envolve o conceito.

Na sequência, é realizado o estudo das propriedades gerais, identificando a transformação do modelo da relação; para que, em seguida, se busque a concretização das propriedades gerais na resolução das tarefas particulares. Posto isso, o aluno terá o controle das ações anteriores, para usar em condições particulares e, por fim, realizar uma avaliação da aprendizagem em todas as ações refletindo sobre elas. De acordo com Davydov (1988, p. 184), “[...] a ação de avaliação permite determinar se o procedimento geral para a resolução de determinada tarefa de estudo é assimilado (e em que medida) ou não, se o resultado das ações do estudo corresponde (e em que medida) ao seu objetivo final", portanto será analisada a assimilação do aluno em relação ao conceito estudado.

Essas ações propostas por Davydov (1988) trazem algo que ultrapassa a forma de organização presente ainda hoje nas escolas que caracterizam a perspectiva tradicional de ensino, focando as ações em apenas uma ou duas funções mentais superiores nem sempre as mais relevantes e indicativas de aprendizagem. A escola deve direcionar o ensino a apropriação do conhecimento científico, buscando que o aluno compreenda de fato o conceito, desenvolva o pensamento teórico e eleve o potencial humano possibilitando a ele estar em constante na atividade enquanto aprende.

\section{CONCLUSÕES}

Por meio dos estudos de Davydov (1982), constatamos que a multiplicação é um conceito fundamental da matemática, que ainda é ensinado de forma empírica nas escolas, o que faz com que a aprendizagem se torne limitada e baseada apenas na repetição e na memorização de dados numéricos. Para mudar essa realidade, é necessário estudar os princípios e nexos que envolvem os conceitos, organizando novas estratégias didáticas. Diante disso, o autor propõe um ensino que possibilite ao aluno a compreensão do conceito por meio da generalização, com 0 intuito de formar o pensamento teórico, finalidade da educação escolar.

Esse ensino pode ser organizado por meio da AOE e de ações de estudo que se concretizam em tarefas propostas pelo professor, 
que podem possibilitar que o aluno entre em atividade de estudo e, assim, compreenda o conceito de multiplicação. Estar em atividade implica em assumir uma ação investigativa diante dos conceitos apreendidos e possibilita a criação de condições adequadas para o processo de ensino e aprendizagem do conceito multiplicativo.

De acordo com o exposto, de acordo com os pressupostos da Teoria Histórico-Cultural e da Atividade Orientadora de Ensino, a escola precisa direcionar suas ações a formação do pensamento teórico. Esperamos com esta pesquisa auxiliar na compreensão do conceito de multiplicação reconhecendo que quando ensinamos de fato os conceitos estamos pensando o fenômeno educativo como propulsor do pleno desenvolvimento humano. Acreditamos na necessidade de que novas pesquisas sejam feitas com essa problemática, buscando assegurar um movimento de continuidade que auxilie na sistematização de proposições didáticas para o trabalho em sala de aula com esse conceito.

\section{REFERÊNCIAS}

CATANANTE, I. T; ARAUJO, E. S. Os limites do cotidiano no ensino da matemática para a formação de conceitos científicos. Poiésis, Tubarão, Volume Especial, p. 45 - 63, Jan/Jun. $2014 . \quad$ Disponível em: https://doi.org/10.19177/prppge.v8e0201445-63 Acesso em: 21 set. 2019.

DAMAZIO, A, ROSA, J. E. Educação matemática: possibilidades de uma tendência históricocultural. Espaço Pedagógico. v.20, n.1, p.33-53. $2013 . \quad$ Disponível em: https://doi.org/10.5335/rep.2013.3506. Acesso em: 21 set. 2019.

DAVYDOV, V. V. Análisis de los principios didáticos de la escuela tradicional y posibles principios de enseñanza en el futuro próximo. In: SHUARE, M. La psicologia Evolutiva y pedagógica en la URSS. p. 143-155, 1987.

DAVYDOV, V. V. La actividad de estudio en la edad escolar inicial. In: DAVYDOV, V. V. La enseñanza escolar y el desarrollo psíquico: investigación teórica y experimental. Trad. Marta Shuare, Moscú: Editorial Progreso, 1988, p.158191.

DAVYDOV, V. V. Tipos de generalización en la enseñanza. 3. ed. Habana: Editorial Pueblo y Educación, 1982.

FIORENTINI, D. Alguns modos de ver e conceber o ensino da matemática no Brasil. Revista Zetetikê. Ano 3, n. 4, p.1-38. 1995.

GALDINO, A. P. S. O conhecimento matemático de estudantes do 3ㅇ ano do ensino fundamental sobre o conceito de multiplicação: um estudo com base na teoria histórico-cultural. 2016. Dissertação (Mestrado em Educação) Universidade do Sul de Santa Catarina, Tubarão 2016.

HOBOLD, E. S. F; ROSA, J. E. O ensino da tabuada no contexto das ações de estudo propostas por Davýdov e colaboradores. Revista Brasileira de Educação. V. 22, n. 71, p. 1-30. 2017. Disponível em: $\quad$ https://doi.org/10.1590/s141324782017227158. Acesso em: 21 set. 2019.

MOURA, M. O. Matemática na infância. In: MIGUEIS, M. R.; AZEVEDO, M.G. Educação matemática na infância: abordagens e desafios. Serzedo, Vila Nova de Gaia: Gaialivro, 2007. p. 3964.

MOURA, M. O. A atividade de ensino como ação formadora. In: CASTRO, A. D. E. CARVALHO, A.M P. Ensinar a Ensinar. São Paulo: Pioneira, 2001, p. 143- 162.

MOURA, M. O. et al. Educação Matemática nos anos iniciais do ensino fundamental: princípios e práticas da organização do ensino. In: Encontro Nacional de Didática e Práticas de Ensino, 16., 2012, Campinas. Anais [...]. Campinas: UNICAMP, 2012, p.002478-002489.

OLIVEIRA, V. S. et al. Educação e Desenvolvimento do Psiquismo: uma análise Histórico-Cultural acerca dessa correlação. Colloquium Humanarum, v. 14, n. 1, p. 60-66, jan/mar 2017. Disponível em: https://doi.org/10.5747/ch.2017.v14.n1.h294. Acesso em: 21 set. 2019.

ORTIGÃO, M. I. R; JUNIOR, C. A. A. Avaliação das aprendizagens em matemática: repensando processos meritocráticos. In: Encontro Nacional de Educação Matemática, 12., 2016, São Paulo. Anais [...]. São Paulo, 2016, p.1-8. 
ROSA, J. E. Proposições de Davydov para o ensino de matemática no primeiro não escolar: inter-relações dos sistemas de significações numéricas. 2012. Tese (doutorado) Universidade Federal do Paraná, Setor de Ciências Humanas, Curso de Doutorado em Educação, Curitiba, 2012.

ROSA, J. E; HOBOLD, E. S. F. O modelo da tabuada na proposição davydoviana. Ciência e Educação, Bauru. V. 23, n. 2, p. 437-454. 2017. Disponível em: $\quad$ https://doi.org/10.1590/1516731320170020010. Acesso em: 21 set. 2019.

ROSA, J. E. et al. Fundamentos da Teoria Histórico-Cultural que orientam o modo de organização do ensino de matemática no contexto da atividade orientadora e da proposição davydoviana. In: Encontro Nacional da Educação Matemática, 12. 2016, São Paulo. Anais [...]. São Paulo, 2016, p.1-8. 